Accepted manuscript (c) <2020>. This manuscript version is made available under the CC-BY-NC-ND 4.0 license http://creativecommons.org/licenses/by-nc-nd/4.0/

Published in: X-ray Spectrometry 44 93-98 (2015)

\title{
X-ray spectrometry and imaging for ancient administrative handwritten documents
}

Fauzia Albertin, ${ }^{a}$ Alberto Astolfo, ${ }^{b}$ Marco Stampanoni, ${ }^{b, c}$ Eva Peccenini, ${ }^{d, e}$ Yeukuang Hwu, ${ }^{f}$ Frédéric Kaplang and Giorgio Margaritondo ${ }^{a}$

a Faculté des Sciences de Base, Ecole Polytechnique Fédérale de Lausanne (EPFL), Lausanne, Switzerland b Swiss Light Source, Paul Scherrer Institute, Villigen, Switzerland

c Institute. for Biomedical Engineering, ETHZ, Zurich, Switzerland

d Museo Storico della Fisica e Centro Studi e Ricerche 'E.Fermi', Roma, Italy e Department of Physics, University of Bologna, Bologna, Italy

$\mathrm{f}$ Institute of Physics, Academia Sinica, Taipei, Taiwan

g Laboratoire d'humanités digitales, Ecole Polytechnique Fédérale de Lausanne (EPFL), Lausanne, Switzerland

\begin{abstract}
'Venice Time Machine' is an international program whose objective is transforming the 'Archivio di Stato' - 80 km of archival records documenting every aspect of 1000 years of Venetian history - into an open-access digital information bank. Our study is part of this project: We are exploring new, faster, and safer ways to digitalize manuscripts, without opening them, using X-ray tomography. A fundamental issue is the chemistry of the inks used for administrative documents: Contrary to pieces of high artistic or historical value, for such items, the composition is scarcely documented. We used X-ray fluorescence to investigate the inks of four Italian ordinary handwritten documents from the 15th to the 17th century. The results were correlated to X-ray images acquired with different techniques. In most cases, iron detected in the 'iron gall' inks produces image absorption contrast suitable for tomography reconstruction, allowing computer extraction of handwriting information from sets of projections. When absorption is too low, differential phase contrast imaging can reveal the characters from the substrate morphology.
\end{abstract}

\section{Introduction}

The present study is part of the Venice Time Machine (VTM) project [1] involving the Ecole Polytechnique Fédérale de Lausanne, the University Ca' Foscari of Venice, and the 'Archivio di Stato' in Venice. The project will unlock and transform in a digital data repository the Archivio documents, spanning over ten centuries and documenting every aspect of the Venetian Empire. However, the sheer mass of data is a problem: VTM should digitalize and decipher the entire collection in 10-20 years.

We are specifically exploring new ways to facilitate and accelerate this task. Using X-ray tomography to computer-extracted page-by-page information from sets of projection images could make the virtual 'reading' of manuscripts possible, rapidly and noninvasively. The raw 
data can indeed be obtained without opening or manipulating the manuscripts, reducing the risk of damage and speeding up the process. To explore this possibility, we performed an imaging test with a synchrotron source, in preparation for future in situ imaging in the Archivio using conventional X-ray sources.

For many centuries, two main groups of inks were used, based on iron or on carbon. X-ray absorption imaging is made possible for the first group by the heavy element content of the inks generically denominated 'iron gall'. On the contrary, X-ray absorption contrast is weak for carbon-based inks, although imaging could still be possible with alternate contrast mechanisms.

The name 'iron gall' actually corresponds to a wide variety of ingredients and recipes [2]. As expected, there is a direct correlation between the ink iron content and the quality of X-ray attenuation contrast images.

Thus, a fundamental background issue is the chemistry of the inks used for administrative documents (as opposed to pieces of high artistic or historical value); for such items, the actual composition is not well documented. Furthermore, the Archivio di Stato collection spans many centuries and includes a wide variety of documents such as ship records, notary papers, work tax items, commercial transactions, and demographic accounts. Inevitable fluctuations in the ink recipes are expected: They are among the subjects of our present study.

Our approach is founded on several pioneering studies exploiting X-ray and, in some cases, synchrotron techniques for cultural heritage issues [3-7] and in particular for manuscripts [815]. Synchrotron $X$-ray fluorescence $(X R F)$ was notably used to retrieve 'lost' text from the 'Archimedes Palimpsest' [16], and top-level programs were launched by W. B Seales of the University of Kentucky [17] and by G. R. Davis of the Queen Mary University of London to 'read' ancient manuscripts [18,19]. Using X-ray tomography, the authors of Baumann et al. [17] and Mills et al. [18] virtually 'unrolled' scrolls producing flat readable images and assessing radiation damage. These projects demonstrated the great potential of X-ray techniques in the study of cultural heritage items and in particular of manuscripts.

In the present article, we discuss results on four specimens from our private collection. These specimens were selected because they correspond to the characteristics of part of the Archivio collection as far as the type of manuscript, the writing support, and the period are concerned; however, having no historical value, they did not pose problems of potential damage as the Archivio specimens.

The supports included paper and parchment, and the time period ranged from the 15th to the 17th century. We analyzed a religious parchment from the 15th century (hereafter, P1400), two handwritten letters from 1566 and 1664 (M1566 and M1664), and a simulated 'volume' created by stacking eight fragments of a 1679 commercial document (M1679).

XRF results demonstrated that black iron gall inks were used for all these documents and that the chemical composition is basically consistent with the historical records for the corresponding centuries[2]. However, the relative amount of iron changes drastically between different document areas and from manuscript to manuscript, with no clear timerelated trend. 
In the best situations, the ink iron content was high and the X-ray attenuation image quality was good enough to perform tomographic reconstruction. This was specifically tested for the phantom 'volume' M1679.

In the worst cases, even simple character detection is a problem - and the properties of synchrotron X-ray sources become very helpful. We indeed exploited its spatial coherence [20] to take images with refractive index contrast. As indicated by the extensive experience [21] with other types of specimens, this could allow the recognition of faded out or very weak characters.

\section{Chemistry of ancient inks}

For many centuries, Europeans used the previously mentioned 'iron gall' formula, a name suggesting the presence of iron. The basic fabrication process was the reaction of an acid with an iron compound. The most common procedure involved tannic acid $\left(\mathrm{C}_{76} \mathrm{H}_{52} \mathrm{O}_{46}\right)$ and iron sulfate $\left(\mathrm{FeSO}_{4}\right)$ in rainwater, white wine, or vinegar [22,23].

Tannic acid was obtained from plants, typically the 'galls' growing on trees after parasite attacks (e.g., by gall wasps), and iron sulfate was 'green vitriol' from mines. The reaction produced by oxygen exposure the black pigment ferrotannate.

In addition, the ink contained a water-soluble binder, most commonly gum Arabic - a product of trees, e.g. acacia, rich in polysaccharides and glycoproteins. The main component of gum Arabica is arabin [22,23], a compound of calcium and of the Arabic acid.

The basic composition and fabrication procedure of iron gall inks corresponded in reality to a broad variety of recipes and ingredients such as rainwater, beer, wine, and so on. The fluctuations of the recipes were primarily a consequence of the local preparation of inks by artisans (mostly monks) or the writers themselves.

One important feature of iron gall inks is their evolution with time. Only a small quantity of black pigment is developed using oxygen present in the solution: Most is given by atmospheric oxygen exposure after the writing. The process continues for a long time, resulting in a progressive corrosion of the substrate [22,23].

Our results are consistent with the previous facts. Figure 1 shows XRF spectra for the four analyzed specimens, revealing not only iron but also fluctuations in the iron content.

The spectra were acquired using under same operation conditions with a portable $\mu$-XRF spectrometer ARTAX (Model 200, Brucker) equipped with a Mo anode $\left(1.2 \times 0.1-\mathrm{mm}^{2}\right.$ focal spot size) and a Peltier cooled silicon windowless drift detector ( $<150-e V$ resolution for $\mathrm{Mn}$ $\mathrm{Ka}$ emission). The incoming beam and the detector were at $45^{\circ}$ with respect to the sample normal; the instrument operated under He flux, at $15 \mathrm{kV}$, at $1500 \mu \mathrm{A}$, and with 180-s exposure time. The beam collimator size was $200 \mu \mathrm{m}$.

The specimens were mounted on plastic sponge substrates. Different areas were analyzed for each manuscript, including the substrate, the paper, and several ink areas. The substrate spectra exhibited no features that could affect our analysis. The ink spectra were corrected by subtracting those of the paper area. Although a full quantitative analysis is not possible, 
this correction and the use of the same geometry, voltage, and current conditions for different specimens allowed reasonable comparisons.

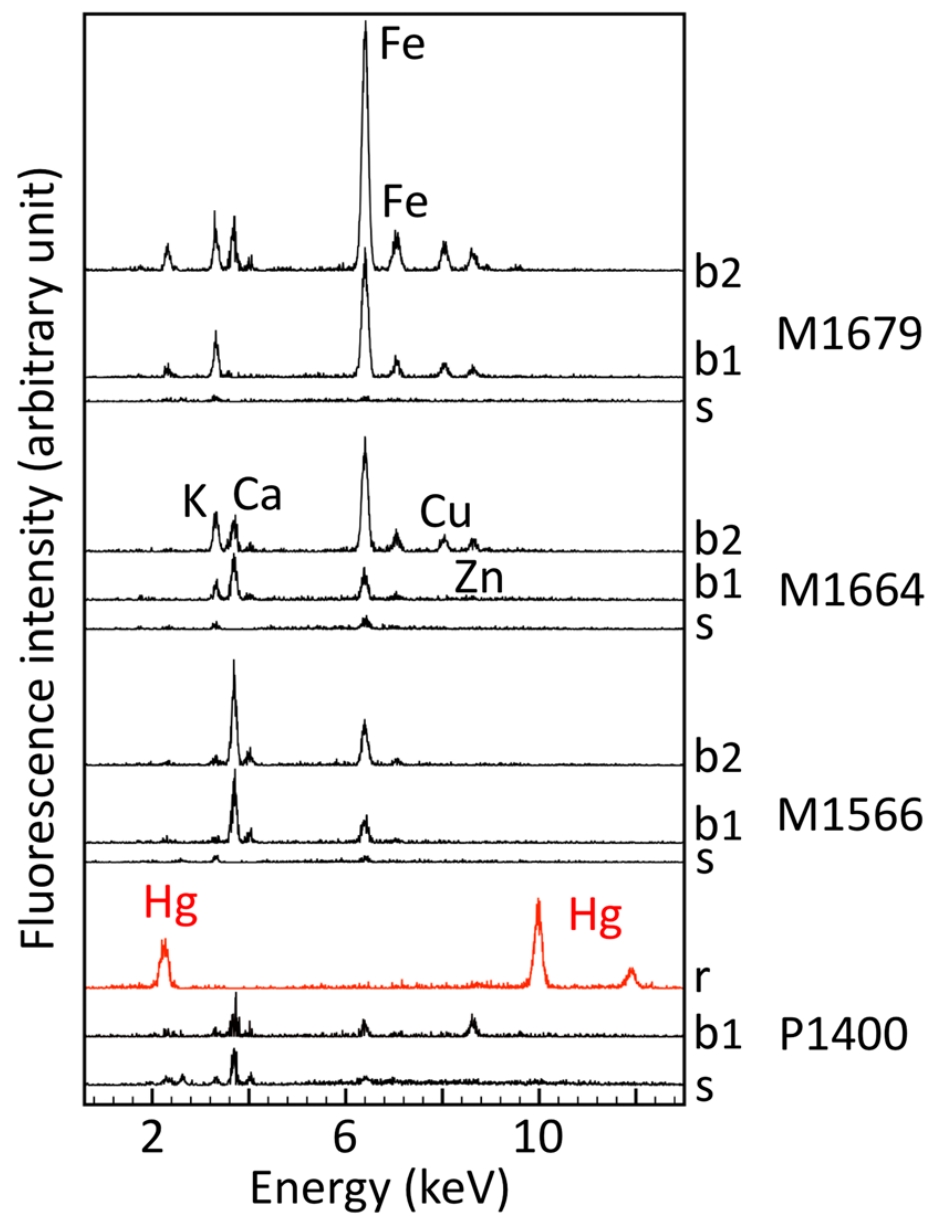

Figure 1. XRF spectra for our four specimens (right-hand-side labels). For each specimen, we report spectra from the substrate (s) and from different black ink and red ink areas (b1, b2, and r).

All spectra revealed Fe in the black ink areas confirming the widespread use of iron gall inks not only for important writings but also for 'everyday' manuscripts. At the same time, they reveal large fluctuations in iron content, not only from manuscript to manuscript but also in the same specimen. In part, the latter effect could have been caused by the handwriting process itself, e.g. the variations in time of the rate of ink release by the pen.

In addition to black ink, ancient manuscripts, in particular those containing important writings, could also use colored inks. This is the case of the P1400 specimen: While the black characters were written with an iron gall ink, the red characters were written with an ink containing mercury (a cinnabar recipe). The $\mathrm{Hg}$ content can be seen in the corresponding spectrum of Fig. 1.

In iron gall inks, other elements were found besides Fe, like calcium. In some cases, we detected $\mathrm{Ca}$ in the ink, consistent with the hypothesis of the use of gum Arabic as the binder. In the P1400 specimen, Ca was found in the substrate, presumably because of chalk used during support preparation [24]. As shown in Fig. 1, other elements attributed to vitriol impurities were found in the black inks, including $\mathrm{K}, \mathrm{Cu}$, and $\mathrm{Zn}$. 


\section{X-ray imaging}

To investigate manuscripts, we used different $\mathrm{X}$-ray imaging techniques: attenuation, phase contrast, differential phase contrast (DPC), and tomography; a detailed descript of these methods can be found, for example, in Margaritondo and Hwu [25] and Weitkamp et al.[26] In short, phase contrast is based on the refraction index rather than on the absorption coefficient; DPC yields images based on the two previous contrast mechanisms plus scattering contrast, and tomography produces threedimensional pictures starting from large sets of projection images. The different contrast mechanisms are complementary and reveal different types of features.

The Fe content of the black inks correlates well with the X-ray attenuation contrast, as shown in Figs 2 and 3(a) for the specimens M1566, M1664, and P1400. The area shown corresponds to the patchwork of images, two for Fig. 2 and nine for Fig. 3(a), each corresponding to the field of view of the instrument. The attenuation images were acquired at the Tomographic Microscopy and Coherent Radiology Experiments beamline [27] of the Swiss Light Source, Paul Scherrer Institute. For the pictures of Fig. 2, we used a photon energy value of $15 \mathrm{keV}$ (which empirically optimized the contrast), a storage ring current of $400 \mathrm{~mA}$ (top-up mode), the free-propagation operation mode for imaging (with a chargecoupled device windowless detector of $2560 \times 1060$ pixels and a pixel size of $6.5 \mu \mathrm{m}$, a 20$\mu \mathrm{m}$-thick LA : Ce scintillator layer). All raw images were processed by dark current and fullfield corrections.

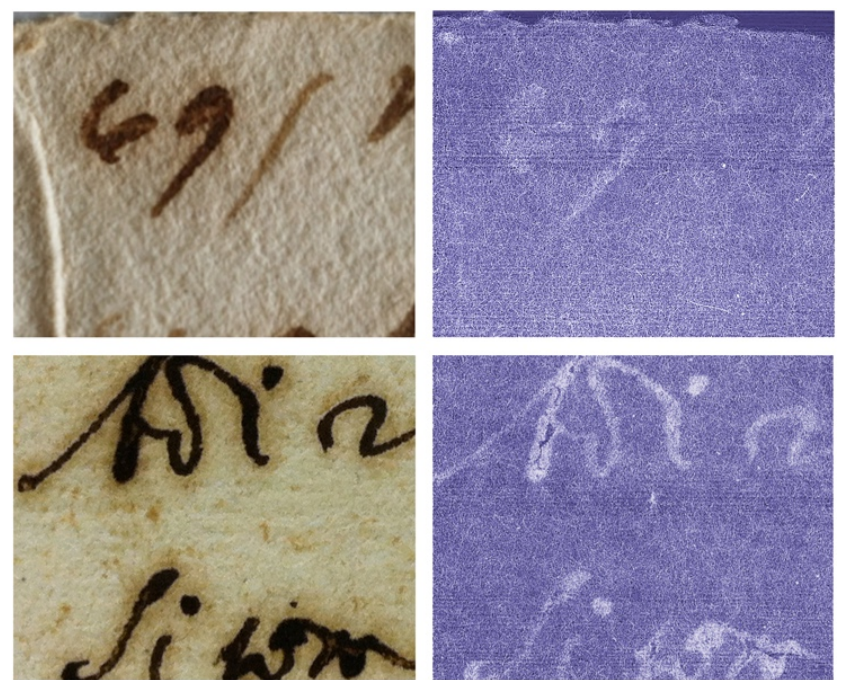

Figure 2. X-ray attenuation contrast $18 \times 14-\mathrm{mm}^{2}$ images for the M1566 (top) and M1664 specimens.

With the adopted sample-detector geometry, these images actually reflect both attenuation and phase contrast: The first prevails in the ink areas, whereas the second is primarily visible in the microscopic substrate features (paper fibers). This is because of the weak absorption in the latter.

The comparison of Figs 1 and 2 is quite straightforward: When the black link contains a high amount of $\mathrm{Fe}$ (M1664 specimen), the attenuation contrast yields easily visible characters. On the contrary, with weak iron content (M1566), the characters are faint and difficult to identify. 
In the cases of low iron content, as for the black ink of the P1400 specimen, a different Xray imaging technique was tested. The pictures in panels $a$ and $b$ shown in Fig. 3 were taken using the same storage ring current and the detector quoted previously for Fig. 2 but a photon energy value of $25 \mathrm{keV}$ and a different imaging mode: DPC[26].
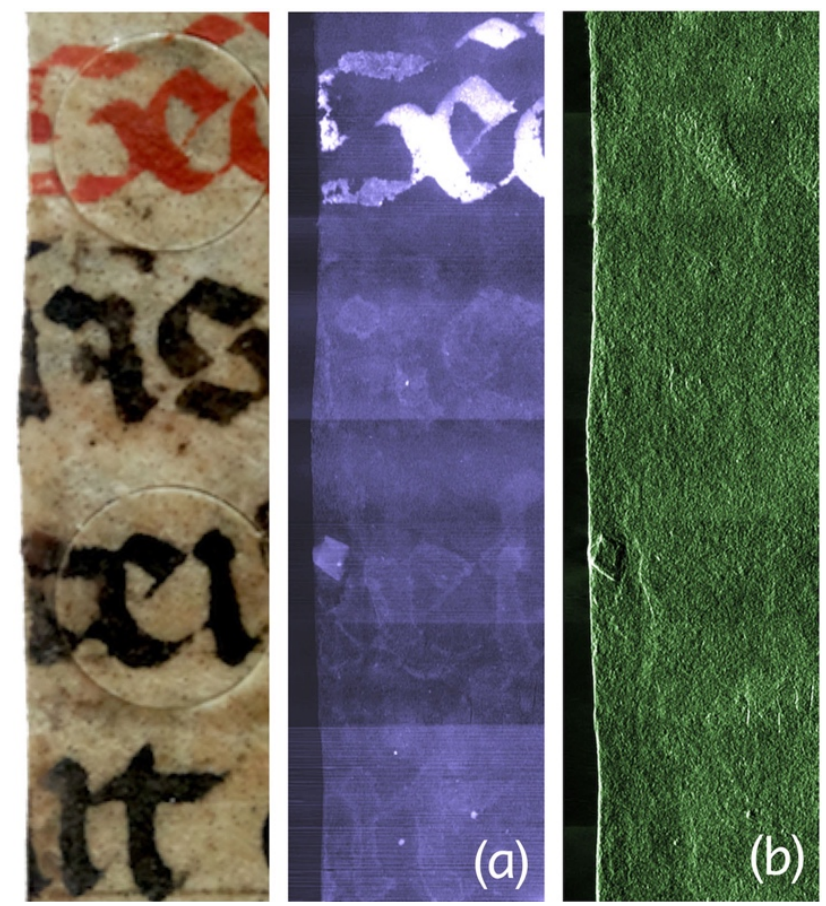

Figure 3. Left: visible photographs of part of the P1400 specimen. Right: absorption contrast (a) and refraction contrast $(b)$ radiographs of the same area. Field of view: $18 \times 63 \mathrm{~mm}^{2}$.

With suitable digit-by-digit computer processing, the raw DPC images yield different pictures corresponding to different contrast mechanisms. In particular, they yield absorption contrast and refraction contrast pictures like Fig. 3(a) and (b). Note the excellent attenuation contrast of Hg-containing red characters, whereas the contrast for black letters is weak, in agreement with the low iron content.

The important point about the refraction image of Fig. 3(b) is that it reflects the local specimen morphology (the parchment topography) rather than only its chemical composition. We can argue that what we see in this figure corresponds to the substrate morphology as modified by the writing process or by the ink-substrate interaction. This could be used to detect a character when the attenuation contrast would only give a very faint picture.

\section{Tomography}

In addition to projection imaging by attenuation or refraction, we also tested the feasibility of tomographic reconstruction. Positive results are shown in Fig. 4 for the M1679 specimen, the artificial 'volume' of eight $0.8-\mathrm{cm}$ diameter fragments. We took a set of $10^{3}$ projection images at equally spaced angles over a $\pi$ rad range, using 15-keV photons and a time exposure of $10 \mathrm{~ms}$ per projection.

The high ink iron content of the specimen (Fig. 1) gives a good absorption contrast, sufficient indeed to perform tomographic reconstruction. In Fig. 4(b), we show a three-dimensional 
reconstructed side view: Note the thin ink layers (the clear regions) corresponding to the characters. The spatial resolution (determined by the $6.5-\mu \mathrm{m}$ detector pixel) is largely sufficient not only to distinguish different pages but also to determine if the writing is in the front or in the back of each 'page'. We also show in Fig. 4(c) and (d) the comparison of a visible picture of one 'page' and the corresponding tomographically reconstructed image. Note the perfectly recognizable character.
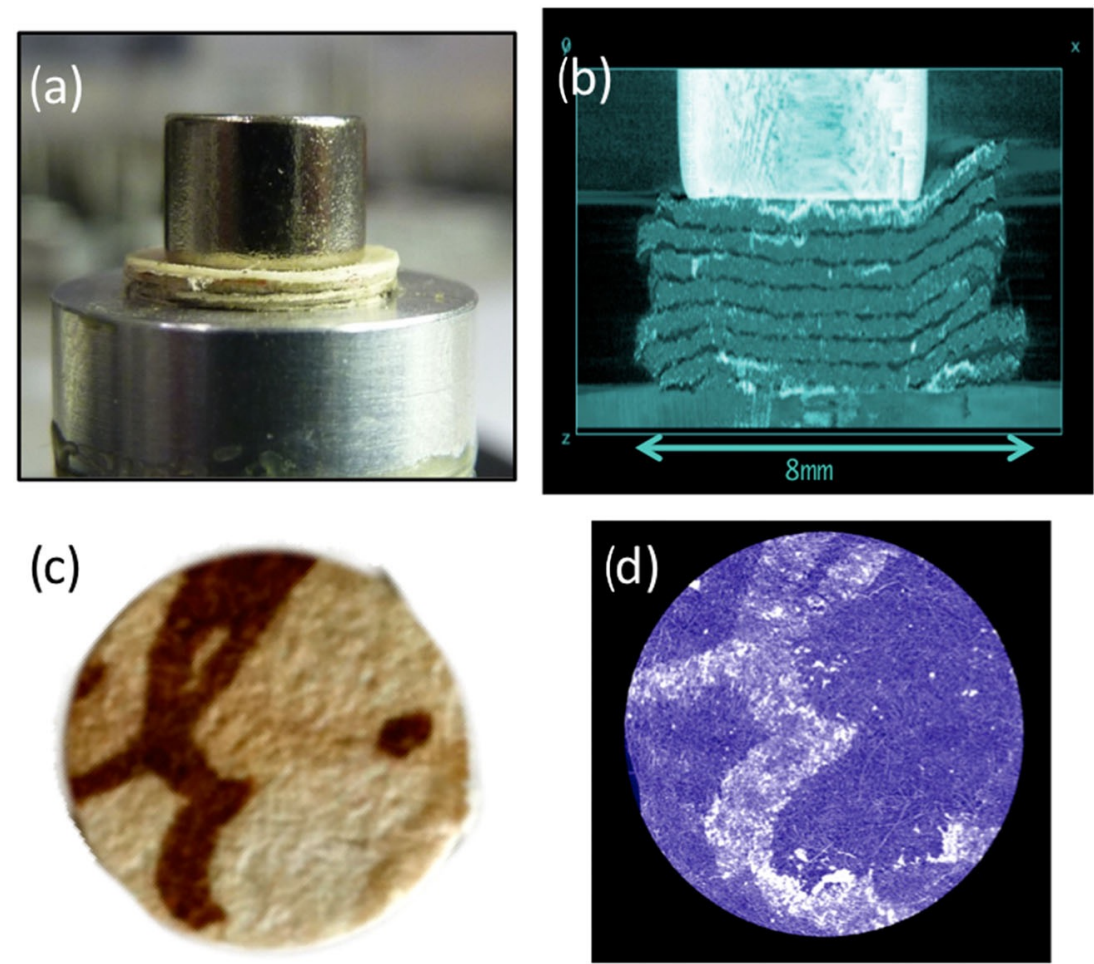

Figure 4. Tomography results for the M1679 specimen. (a) Visible picture with a side view of the stack of 'pages' held in place by a magnet holder, (b) three- dimensional reconstructed image showing the 'pages' with ink visible over them, (c) visible photograph, and (d) tomographically reconstructed picture of a character in one of the 'pages' (diameter $8 \mathrm{~mm}$ ). The color scale reveals in panel $\mathrm{d}$ the character as a clear area.

\section{Conclusions}

Our tests are consistent with the common use of black iron gall inks for administrative and private documents over different centuries, in most cases with iron content sufficient for character detection by X-ray attenuation. We also verified that the corresponding image quality is suitable for advanced tomographic reconstruction. These conclusions open the way to a new strategy for information harvesting from ancient documents - alternate to page-by-page taking of visible pictures. A single tomographic set could yield the same information more rapidly and with minimized interaction with the document. However, in some instances, the iron content is too weak. Our preliminary tests indicate that these cases can be potentially handled with refraction images.

Each projection image set $\left(10^{3}\right.$ images), potentially containing full three-dimensional information, was taken in 10 s (albeit for small samples). This suggests a substantial acceleration with respect to page-by-page approaches. However, many problems remain to be solved along the path to the real applications of the technique. In particular, a crucial future step will be the implementation of the technique with conventional X-ray sources, to perform massive document investigations in situ, without transporting them. The 
corresponding increase in data-taking time must be evaluated with practical tests; the margin of flexibility revealed by our synchrotron present studies is encouraging in that sense. On the positive side, throughout our tests, we found no evidence whatsoever of radiation damage, consistent with the results of Patten et al. [19]

Note that the attenuation and refraction images carry a wealth of information besides the written characters: They can reveal details of the ink-substrate interaction, which in many cases leads to corrosion and deterioration.

\section{Acknowledgements}

The authors are grateful to Patrick Aebischer for his leadership of the VTM project, to the Verbantiqua bookstore (www.copernicum. it) for their generous donation of antique manuscript specimens, to the staff of the Swiss Light Source, Paul Scherrer Institute, for their outstanding technical support, and to Ferruccio Petrucci for his assistance in the chemical analysis and for several stimulating discussions. The research was supported by the Fonds National Suisse pour la Recherche Scientifique, the VTM Project, and the Center for Biomedical Imaging.

\section{References}

[1] F. Kaplan, How to build an information time machine: Venice time machine. Technical report, EPFL, Digital Humanities Lab, 2013. http:// dhvenice.eu/

[2] Yale University Library Special Collections Conservator Unit, Preservation Department. Medieval Manuscripts, Some Ink and Pigment Recipes. Yale University, 2012.

[3] K. Janssens. Synchrotron radiation in art and archaeology. J. Anal. At. Spectrom. 2011, 26, 883-884.

[4] D. Creagh, D. Bradley, Physical Techniques in the Study of Art, Archaeology and Cultural Heritage, Elsevier, Amsterdam, 2007.

[5] L. Caforio, M. E. Fedi, P. A. Mando, F. Minarelli, E. Peccenini, V. Pellicori, F. C. Petrucci, P. Schwartzbaum, F. Taccetti, Discovering forgeries of modern art by the $C^{14}$ bomb peak. Eur. Phys. J. Plus 2014, 129, 1-5.

[6] J. Dik, K. Janssen, G. Van Der Snickt, L. Van Der Loeff, K. Rickers, M. Cotta. Visualization of a lost painting by Vincent van Gogh using synchrotron radiation based X-ray fluorescence elemental mapping. Anal. Chem. 2008, 80, 6436-6442.

[7] M. P. Morigi,F. Casali, M. Bettuzzi, R. Brancaccio, V. D'Errico. Application of X-ray computed tomography to cultural heritage diagnostics. Appl. Phys. A 2010, 100, 653-661.

[8] P. Del Carmine, L. Giuntini, W. Hooper, F. Lucarelli, P. A. Mandò. Further results from PIXE analysis of inks in Galileo notes on motion. Nucl Instrum. Meth. B 1996, 113, 354-356.

[9] F. Lucarelli, P. A. Mandò. Recent applications to the study of ancient inks with external PIXE facility. Nucl. Instrum. Meth. B 1996, 109, 644-652.

[10] A. Mozir, L. Gonzales, I. Kralj Cigic, T. J. Wess, I. Rabin, O. Hahn, M. Strlic. A study of degradation of historic parchment using small-angle X-ray scattering, synchrotron-IR and multivariate data analysis. Anal. Bioanal. Chem. 2012, 402, 1559-1566.

[11] W. Faubel, S. Staub, R. Simon, S. Heissler, A. Pataki, G. Banik. Nondestructive analysis for the investigation of decomposition phenomena of historical manuscripts and prints. Spectrochim Acta B 2007, 62, 669-676.

[12] C. J. Kennedy, J. C. Hiller, D. Lammie, M. Drakopoulos, M. Vest, M. Cooper, W. P. Adderley, T. J. Wess. Microfocus X-ray diffraction of historical parchment reveals variations in structural features through parchment cross-sections. Nano Lett. 2004, 4, 1373-1380.

[13] J. Gunneweg, A. Adriaens, J. Dik, Holistic Qumran: Trans-disciplinary Research of Qumran and Dead Sea Scrolls, Online publication: Brill, 2010.

[14] B. M. Murphy, M. Cotte, M. Mueller, M. Balla, J. Gunneweg, Degradation of parchment and ink of the Dead Sea Scrolls investigated using synchrotron-based X-ray and infrared microscopy. In Holistic Qumran: Trans-disciplinary Research of Qumran and Dead Sea Scrolls, Online publication: Brill, 2010, pp. 77-98. 
[15] J. Dik, P. Helfen, P. Reischig, J. Blaas, J. Gunneweg, A short note on the application of synchrotron based micro-tomography on the Dead Sea scrolls. In Holistic Qumran: Trans-disciplinary Research of Qumran and Dead Sea Scrolls, Online publication: Brill, 2010, pp. 21-27.

[16] U. Bergmann, X-ray fluorescence imaging of the Archimedes Palimpsest: a technical summary. Technical Summary, Report 100, SLAC, 2000.

[17] R. Baumann, D. C. Porter, W. Brent Seales, The use of micro-CT in the study of archaeological artifacts Proceedings of 9th Int Conf on NDT of Art, 2008.

[18] D. Mills, O. Samko, P. Rosin, K. Thomas, T. Wess, G. R. Davis, Apocalypto: revealing the unreadable. Proc. SPIE, 8506:VIII:85060A, 2012.

[19] K.Patten,L.Gonzales,C.Kennedy,D.Mills,G.R.Davis,T.Wess.Isthere evidence for change to collagen within parchment samples after exposure to an X-ray dose during high contrast X-ray microtomography? A multi technique investigation. Herit. sci. 2013, 1, 1-9.

[20] G. Margaritondo, Elements of Synchrotron Light for Biology, Chemistry, and Medical Research, Oxford, New York, 2002.

[21] Y.Hwu,H.H.Hsieh,M.J.Lu,W.L.Tsai,H.M.Lin,W.C.Goh,B.Lai,J.H.Je, C. K. Kim, D. Y. Noh, H. S. Youn, G. Tromba, G. Margaritondo. Coherence enhanced synchrotron radiology: refraction versus diffraction mechanisms. J. Appl. Phys. 1996, 86, 4613-4618.

[22] G. Smith. The chemistry of historically important black inks, paints and dyes. Chem. Educ. in New Zeland 2009, 12-15.

[23] D. Ruggiero, Gli inchiostri ferrogallici negli archivi e nelle biblioteche. Technical report, Laboratorio di Fisica dell'Istituto per il Restauro e la Conservazione del Patrimonio Archivistico e Librario, 2002.

[24] G. van der Snickt, W. De Nolf, B. Vekemans, K. Janssens. $\mu-X R F / \mu-R S$ vs. SR $\mu$-XRD for pigment identification in illuminated manuscripts. Appl. Phys. A 2008, 92(1), 59-68.

[25] G. Margaritondo, Y. Hwu. The new birth of radiology - more than one century after Röntgen. Nuovo Sag. 2013, 29, 45-58.

[26] T. Weitkamp, A. Diaz, C. David, F. Pfeiffer, M. Stampanoni, P. Cloetens, E. Ziegler. X-ray phase imaging with a grating interferometer. Opt. Express 2005, 13, 6296-6304.

[27] M. Stampanoni, A. Groso, A. Isenegger, G. Mikuljan, Q. Chen, D. Meister, M. Lange, R. Betemps, S. Henein, R. Abela. Tomcat: a beamline for tomographic microscopy and coherent radiology experiments. Synch. Rad. Instr 2007, 1 and 2, 848-851. 